\title{
Towards a Code of Ethics for Gamification at Enterprise
}

\author{
Alimohammad Shahri, Mahmood Hosseini, Keith Phalp, Jacqui Taylor, and Raian Ali \\ Bournemouth University, UK \\ \{ashahri,mhosseini, kphalp, jtaylor,rali\}@bournemouth.ac.uk
}

\begin{abstract}
Gamification is an emerging technique which utilises the "fun theory" mainly to motivate people to change their perception and attitude towards certain subjects. Within enterprises, gamification is used to motivate employees to do their tasks more efficiently and perhaps more enjoyably and sometimes to increase their feeling of being members of the enterprise as a community. While the literature has often emphasised the positive side of gamification, mainly from economic and business perspectives, little emphasis has been paid to the ethical use of gamification within enterprises. In this paper we report an empirical research to explore the ethical aspects of using gamification. We follow a mixed methods approach involving participants who are gamification experts, employees and managers. Our findings show that, for gamification, there is a fine line between being a positive tool to motivate employees and being a source of tension and pressure which could then affect the social and mental well-being within the workplace. This paper will evaluate that dual effect and clarify that fine line.
\end{abstract}

Keywords: Gamification, Ethical Gamification, Well-Being within Enterprise.

\section{Introduction}

Gamification is commonly defined as the use of game design elements in a non-game context [1]. Examples include the use of points and leader-boards for staff in a call centre to reflect the number of calls answered, the issues resolved, the time taken, and the customer satisfaction [2]. Huotari and Hamari [3] emphasise the creation of an added value to the enterprise, e.g. increasing staff engagement and the affordability of a gameful experience, as core elements for gamification. Gamification has been applied in a diversity of domains including education [4], e.g. to increase performance and engagement of students [5], enterprise, e.g. to increase staff and customers loyalty [6], and design, e.g. to encourage sustainable living [7].

The literature on gamification has mainly advertised it as a creative way to increase engagement and motivation while its downside has been overlooked. When badly designed and applied, it could be a genuine harm for social and mental well-being within the workplace. Stakeholders' awareness of those issues should be integrated in the development process of gamification and its deployment in an enterprise.

In this paper, we make a start in studying the ethical and professional issues which should be observed when applying gamification within an enterprise. To explore this, we adopt a mixed methods approach [8] consisting of three phases; an exploration phase, a confirmation phase, and a clarification phase. Our results are intended to provide a checklist for system analysts when applying gamification within the workplace and raise awareness of this under-researched side of gamification. 


\section{Study Design}

Fig. 1 1 summarises our research method. The first two stages, the exploration stage and the confirmation and enhancement stage contained a study of further aspects which are not discussed in this paper and relating mainly to the definition of gamification, its stakeholders, fields involved, and good design principles.

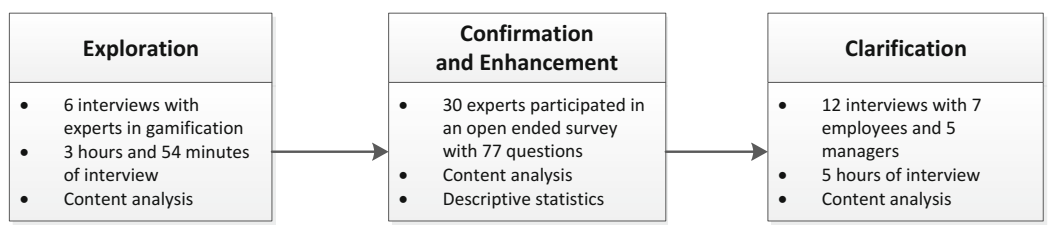

Fig. 1. Research methodology

In the exploration phase, we used interviews, a widely used data gathering tool in qualitative research [9]. Our participants were experts in gamification. In our study, experts were identified based on their constant and influential contribution to the field manifested in peer-reviewed publications. In this phase, we interviewed six experts (four from academia and two from industry). All experts implemented gamification in practice, and three also contributed theoretical frameworks. The experts came from six different countries - UK, South Africa, USA, Portugal, Germany, and Canada. The fields where our experts applied gamification were also diverse and included business, education, human resource development, and creative activities. A content analysis of the answers was conducted by two of the authors and led to 11 main statements.

The confirmation and enhancement phase was survey-based and was designed to confirm and enhance the 11 statements obtained via the first phase. Each statement was converted to a question with a five-point Likert scale reflecting the degree of agreement or disagreement with the statements. A text entry box was also provided for further insights and comments. 42 experts were invited, and 30 of them completed the survey. Our experts worked in various affiliations based in different countries: Germany, Italy, USA, UK, France, Netherlands, Japan, Portugal, China, and Norway. Their fields of expertise included Education, Game Design, Sociology, Modelling and Theory, Economics, Linguistic Annotation, Marketing, Psychology, Enterprise, Ergonomics, HCI, UX, Health, Game Development, Exertion Interfaces, Tourism, Motivational Mechanism, Behavioural Perspective, and Design. The full interview questions and questionnaire can be found on http://goo.gl/wBZtiR

The clarification phase was designed to clarify the findings of the first two phases from the perspective of users. We looked for diversity in users' roles in the enterprise and interviewed 12 people, five who typically had a managerial role and seven who were other employees. We selected participants who were familiar with gamification and who use computers as a main medium for their jobs. Diversity in age, gender and work domain was also ensured, including nine males and three females, and their age ranged from 30 to 58 years old. The full list of interview questions can be found on http://goo.gl/p15w1j. 


\section{Results}

By analysing the answers from the eight expert interviewees in the exploration phase, we deduced the below 11 statements on the relation between gamification and ethics. In the confirmation and enhancement phase, these statements were confirmed by 30 experts using a five-point Likert scale. The percentages are shown after each statement (SA: Strongly Agree, A: Agree, N: Neutral, D: Disagree, SD: Strongly Disagree).

1. Gamification can lead to tense relationship amongst colleagues, e.g. when applying a leader-board (SA: 30\%, A: 43\%, N: 17\%, D: 7\%, SD: 3\%)

2. Gamification could lead to rating people and creating classes, i.e. more pressure and impact on the equity principles (SA: 17\%, A: 47\%, N: 20\%, D: 17\%, SD: 0\%)

3. Gamification can create tension on the person, e.g. it could be seen as a monitoring system on how well a person is performing (SA: 27\%, A: 50\%, N: 7\%, D: $17 \%$, SD: $0 \%$ )

4. Gamification captures a lot of personal data, e.g. about performance. Privacy policies and data protection need to be augmented by ethical awareness (SA: 20\%, A: 43\%, N: $27 \%, \mathbf{D}: 7 \%$, SD: $3 \%$ )

5. Gamification can lead to exposure of information users are not necessarily willing to expose, e.g. listing the top 10 performers reveals if someone was never a top performer. (SA: 23\%, A: 47\%, N: 20\%, D: 10\%, SD: 0\%)

6. Freedom of Information: Users' ability to see what is stored about them is an ethical issue (SA: $43 \%, \mathbf{A :}$ 37\%, N: 13\%, D: 7\%, SD: 0\%)

7. Gamification, in certain cases, could mean trying to get from people more than what their job requires, i.e. using gamification as an exploitation-ware (SA: 23\%, A: $40 \%, \mathbf{N}: 13 \%, \mathbf{D :} 23 \%, \mathbf{S D}: 0 \%)$

8. The desire for "wining the reward" could drive some users to overlook how data is gathered and to whom it is exposed. This makes some users, at times, vulnerable (SA: $17 \%, \mathbf{A :} 47 \%, \mathbf{N}: 20 \%$, D: $17 \%$, SD: $0 \%$ )

9. Ethics in gamification could be seen analogous to those in marketing, i.e. gamification could make some tasks attractive to users who would not ethically like to perform without gamification (SA: 10\%, A: 43\%, N: 40\%, D: 7\%, SD: 0\%)

10. Ethics should be seen case by case and even at the level of individual users, e.g. the same game mechanic for the same task may be seen differently from ethical perspective according to the user (SA: 14\%, A: 59\%, N: 24\%, D: 3\%, SD: 0\%)

11. Gamification ethics are highly dependent on the norms and culture of the organisation (SA: 37\%, A: 50\%, N: 10\%, D: 3\%, SD: 0\%)

The experts' comments in these open-ended survey questions were also analysed to obtain further insights which will be reported, together with those obtained from the clarification phase with employees and managers, in the subsequent sections. The analysis in these two phases led to grouping the above statements into five categories:

- Gamification and tension at workplace $(1,2,3)$

- Gamification as a monitoring mechanism $(3,4)$

- Gamification and privacy $(4,5,6)$

- Using gamification as "exploitation-ware" $(7,8)$

- Gamification and its relation to personal and cultural values $(9,10,11)$ 


\subsection{Gamification and Tension at Workplace}

Nature of the Working Environment. The results show that implementing gamification in a collaborative environment will have a negative impact, since it creates an unnatural competition and thus tension, affecting the relationship amongst employees who will be heavily comparing themselves with each other, which is not the best practice for collaborative projects. In environments where competition is natural, e.g. bonuses to the best performers in a call centre, this effect of gamification could be acceptable.

Tasks. A main attribute of a business task, which matters here, is whether it is a collaborative or a competitive task regardless of the nature of the work environment. In addition, gamification seems to suit tasks which are with a measurable outcome, objective and done separately by a group of people. If the tasks can be measured, for example answering clients in a sales environment, then gamifying the task is unlikely to create or increase tension since it will provide employees with a system that showcases their efforts. Applying gamification for subjective tasks may lead to tension since quantifying the actual effort is usually hard. Applying gamification uniformly on different tasks could lead to more tension as "some may have easier tasks than others".

Age. Participants observed that older generation employees might not like to work in environments where gamification is applied, as it may lead to taking them out of their comfort zone and put them under the pressure of learning a new technology and to know in certain cases how to adjust their work style to get the best of it.

Employee's Personality. The achievements may make some employees arrogant or, in contrast, some employees may start to be helpful and try to train others. Some of them might be competitive and like it, while some "might just get stressed by thinking about it". Extroverts typically like showing their achievements, e.g. sharing their badges, while introverts may find it stressful.

The Management Style. In highly hierarchical and centralised management styles, gamification could lead to stressing people by creating the fear of being questioned frequently. Also, managers could use the collected information to compare employees with each other and create competition for promotions, hence creating tension. In different non-hierarchical environments, however, managers may use the data for improving employees' weaknesses, identifying their skills and finding the best role for them.

Employees' Ranks. Ranking employees via gamification could create tension, "If you appear in the leader-board, you are fine, but if you do not, you may feel depressed". Ranking could also have a negative impact on employees' relationships, decreasing the teamwork since "those who are below their peers will feel pressurised". However, ranking could increase teamwork when it is applied to a team instead of the individuals. 
Clusters Amongst Employees. Employees who are performing similarly could group together and those who are under-performing might be asked to leave the group since they are deteriorating the team score and profile. However, clustering based on gamification is not necessarily negative as "it may create unity in a team, [since] it can group people [with similar talents] together".

Table 1 summarises our findings about the factors that can introduce tension into the working environment and clarify the fine line between questionable use of gamification and the use which is likely to be acceptable from ethical perspectives.

Table 1. Gamification vs. tension at workplace

\begin{tabular}{|l|l|l|}
\hline & Tension-Problematic & Tension-Acceptable \\
\hline Working Environment & Collaborative & Competitive \\
\hline \multirow{4}{*}{ Task } & Not measurable & Measurable \\
\cline { 2 - 3 } & Uniform & Non-uniform \\
\cline { 2 - 3 } & Subjective & Objective \\
\cline { 2 - 3 } & Collaborative & Competitive \\
\hline Age & Older generation & Younger generation \\
\hline \multirow{2}{*}{ Personality } & Non-competitive & Competitive \\
\cline { 2 - 3 } & Introverts & Extroverts \\
\hline \multirow{2}{*}{ Management Style } & Comparative appraisal & Individual appraisal \\
\cline { 2 - 3 } & Destructive criticism & Constructive criticism \\
\hline \multirow{2}{*}{ Rank } & Rarely top performing & Regularly top performing \\
\cline { 2 - 3 } & Individual competition & Group collaboration to win \\
\hline Clustering & Fear of being isolated & Talent-based grouping \\
\hline
\end{tabular}

\subsection{Gamification as a Monitoring Mechanism}

Monitoring performance is a common practice within enterprises even without gamification. The difference is that gamification can do that in a very detailed way. It may also capture sensitive personal data, e.g. the analysis of a webcam to deduce an employee's mood and mental status and reflect it on the avatar representing that employee. Monitoring from the perspective of privacy will be discussed in another section.

The Wide Visibility of Employees' Ranks in Enterprises. Leader-boards can be visible to everyone and can be perceived as a monitoring system. Employees may not like that, given that many will not appear as top performers. On the other hand, top performers embrace this feature as they can showcase their progress to other colleagues and especially managers. Top performers are recognised in traditional monitoring and appraisal systems but with a less frequency and visibility than the case with gamification.

Level of Details. Employees may have various productivity levels during working hours, referred to as highs and lows. Gamification can easily collect such details, giving managers the opportunity to know better the working pattern of a certain staff. Employees typically prefer to have control over how they perform the task and to make only 
the final results visible to the managers, not their performance during the process to achieve that. However, when detailed information is captured, employees could feel the pressure to have a constant level of performance.

The Nature of Tasks. Monitoring in general, and fine-grained monitoring in particular, puts pressure on employees when the tasks require creativity and one cannot predict how much time they should take. In addition, gamification-based monitoring may not reflect the true nature of the task. In a sales environment, for example, some of the employees may argue that although they have sold less, thus scoring less, but they had to deal with difficult and time demanding customers. Gamification can capture how much work is done, but is often limited in capturing the quality.

The Management Style. Employees tend to accept gamification as a monitoring system, as long as they are certain that managers would use it to help them improve, without comparing them to others and using it to make them put extra effort with the same pay. Furthermore, gamification can spot a performance problem but cannot interpret it and its context. When managers rely purely on gamification, it may lead to a misinterpretation of what is really happening. Managers should keep a direct contact with the employee to give meaning to the data monitored by gamification.

The Employees' Personality. Gamification could be used as a self-monitoring mechanism for employees who are interested in knowing how well they are performing and who use it to self-motivate themselves. However, personality traits could also play a key role here, as employees who are not genuinely interested in the job and who are "looking for promotions and just want to do the job" will rarely perceive monitoring in such a positive way.

Table 2 summarises our findings about factors related to gamification as a monitoring mechanism and how likely they are to raise issues in the working environment.

Table 2. Monitoring mechanism factors vs. their perception

\begin{tabular}{|l|l|l|}
\hline & Likely to Raise Issues & Likely to be Accepted \\
\hline \multirow{2}{*}{ Rank Visibility } & Not in the top list & In the top list \\
\cline { 2 - 3 } & Frequently shown to all & Occasionally shown to all \\
\hline Level of Details & Fine-grained details & Overall performance \\
\hline \multirow{2}{*}{ Nature of Task } & Creative & Classical \\
\cline { 2 - 3 } & Quality-based & Quantity-based \\
\hline \multirow{2}{*}{ Personality } & No direct contact & Direct contact \\
\cline { 2 - 3 } & $\begin{array}{l}\text { Pressurising for } \\
\text { more profit }\end{array}$ & Improving self-productivity \\
\cline { 2 - 3 } & Doing the task as any job & $\begin{array}{l}\text { Genuinely interested } \\
\text { in the task }\end{array}$ \\
\cline { 2 - 3 } & Moderately ambitious & $\begin{array}{l}\text { Ambitious and } \\
\text { self-motivated }\end{array}$ \\
\hline
\end{tabular}




\subsection{Gamification and Privacy}

As a counterpart of monitoring, privacy is seen as a main concern when using gamification. The concerns were centred on the following categories:

What is Being Stored? The use of gamification to capture work-related information, e.g. how many issues an employee has solved, seems to be acceptable. However, it raises issues when gamification captures personal information, or information which can lead to personal information, e.g. analysing the calls to know the mood of an employee and change the avatar accordingly. Also, the stored information should be objective facts, e.g. storing the time taken to solve an issue, rather than judgements which are subject to different interpretation and deduction methods, e.g. storing that an employee is tired or lazy because of the long time taken.

Who Can See the Information? Employees would not accept that gamification data is widely visible even for data reflecting their unique areas of expertise. This privacy concern is lighter when the data is available to managers who are legally entitled to monitor employees performance. Similarly, privacy concerns are less when data is available to relevant colleagues, especially when working as a team. This needs to be still based on clear organisational rules. Anonymity is also another aspect. Through the use of an anonymised or translucent leader-board, employees will know how well they are doing in comparison to the top performers, still without revealing anyone's identity.

The Employees' Personality. Typically, hard-working, competitive and ambitious employees will be less worried about privacy issues when gamification captures data related to their performance. They may see it an advantage, e.g. when applying for promotions or bonuses. Some others may not like competition and this may not be due to their low performance. They typically tend to be introverts and happy with their positions.

The Right to View Information. Participants agreed that employees should have the right to see what gamification reports capture about them. In essence, under the Freedom of Information Act in some countries, people are legally given this right. However, it stays in the grey area whether employees can also view how exactly the data was processed to infer a judgement which might not be straightforward or even algorithmic in certain cases.

Table 3 summarises our findings about factors related to privacy issues in gamification and how likely they are to raise issues in the working environment.

\subsection{Gamification as "Exploitation-Ware"}

The term "exploitation-ware" refers to the use of gamification to motivate staff to do more than what their job requires [10]. 
Table 3. Privacy vs. employees perception at workplace

\begin{tabular}{|l|l|l|}
\hline & Likely to Raise Issues & Likely to Be Accepted \\
\hline \multirow{4}{*}{ Stored Information } & $\begin{array}{l}\text { Personal, or likely to } \\
\text { lead to infer personal } \\
\text { information }\end{array}$ & Work-related information \\
\cline { 2 - 3 } & Subjective judgement & Objective facts \\
\hline $\begin{array}{l}\text { Information } \\
\text { Accessibility }\end{array}$ & $\begin{array}{l}\text { Public/non-relevant } \\
\text { peers }\end{array}$ & Managers/relevant peers \\
\cline { 2 - 3 } & Real names & $\begin{array}{l}\text { Anonymised or } \\
\text { translucent }\end{array}$ \\
\hline \multirow{2}{*}{ Personality } & Introvert & Extrovert \\
\cline { 2 - 3 } & Non-competitive & Competitive \\
\cline { 2 - 3 } & Ambitious & Happy where they are \\
\hline \multirow{2}{*}{ Right to View Information } & $\begin{array}{l}\text { Actual collected data } \\
\text { and their interpretation } \\
\text { are hidden }\end{array}$ & Both are available \\
\hline
\end{tabular}

The Strategy of Rewarding. The tendency to consider gamification as an "exploitationware" increases when the reward strategy depends on the relative performance of an employee with respect to others rather than being dependent on the individuals' performance progress. An example of this is when the top-ten performers get a higher salary while the rest, who still tried their best, are not rewarded. The design of a tempting reward mechanism which attracts many but can be ultimately achieved only by very few employees is likely to raise exploitation-related issues.

The Nature of Reward. Intangible rewards, such as being in the leader-board, have no "real" costs and could still drive employees to work harder. Intangible rewards could be viewed as an exploitation of the social environment and peer-pressure at an enterprise in order to get more work done without a significant tangible investment.

The Transparency of Rewarding Policy. If managers explain how points will be translated to promotions on an objective basis, this makes gamification more professional. Such translation is sometimes not easy to make and managers tend to do their evaluation of performance on a case by case basis by using their tacit knowledge. Thus, the problem is not solely about transparency but also about the ability to concretise and quantify the rewarding strategy.

The Perception of the Traditional Version of the Rewarding Mechanism. When the underlying reward strategy is ethically accepted, then its automated or gamified version is unlikely to be seen differently. For example, if in certain enterprises a draw conducted to choose one of the top performers to receive a gift is a well-accepted practice in the enterprise, gamifying it will not raise ethical issues. If such a procedure is seen as bringing lottery and gambling to the work environment, then gamifying it would raise similar ethical and professional issues. 
Employees' Personality. Gamification could be seen as an "exploitation-ware" when applied to people who like intangible rewards and value them in an exaggerated way. Those who are obsessed in developing their online reputation would value a nicer avatar more than a salary increase. A similar observation could be made for those who are socially isolated in the traditional world and who try to compensate in the gamification world. Enterprise management should make sure that such exaggerated appreciation of virtual rewards is handled beforehand.

Table 4 summarises our findings regarding the factors related to the perception of gamification as an exploitation-ware.

Table 4. Gamification as exploitation-ware

\begin{tabular}{|l|l|l|}
\hline & Likely to Raise Exploitation Issues & Likely to Reduce Them \\
\hline Rewarding Strategy & $\begin{array}{l}\text { Comparing to others } \\
\text { progress }\end{array}$ & Comparing to self-progress \\
\hline Nature of the Reward & Intangible costs & Tangible costs \\
\hline Policy & Non-transparent, unexplained & Transparent, explained \\
\hline \multirow{2}{*}{ Tasks } & $\begin{array}{l}\text { Non-concrete/ } \\
\text { subjective }\end{array}$ & $\begin{array}{l}\text { Concrete/ } \\
\text { objective }\end{array}$ \\
\hline Underlying Mechanism & Seen negative & Seen acceptable \\
\hline Personality Type & Online "ultras" & Balanced \\
\cline { 2 - 3 } & Looking to compensate online & Balanced \\
\hline
\end{tabular}

\subsection{Gamification vs. Personal and Cultural Values}

Gamification and the desire to win, as an underlying concept, could lead to employees acting against their personal and cultural values. For example, in a call centre, an agent would tolerate the language of an angry customer to get the points of solving the issue.

Value-Sensitive Design. Gamification per se is not a reason for people to behave in a certain manner. It is just a facilitator. However, this should not mean that gamification developers and enterprise managers are exempted from any responsibility when applying it. Gamification, especially for the "digital-native" generation, could be a very attractive medium which facilitates acting against their personal and cultural values just to win the virtual reward. On the other hand, employees should have the freedom of rejecting mechanisms they see against their values. This shows the importance of value-sensitive design [11] of gamification as a kind of information systems.

Sacrificing Quality Standards. Gamification could drive people to do things in a cursory manner. The fear of losing their community recognition, when techniques like ranks and status and leader boards are applied, could be a main reason for that.

Cheating to Win/Survive. The desire to win, and also the fear of the failure, could drive people to cheat and do the tasks in a way which would contradict with their own values. This was observed in [5] through a case study in the education sector. 
The Culture of the Place. The culture of the enterprise and the country where it resides is a key factor. In certain cultures, showing off is seen as a violation of the norms and conventions of acceptable public behaviour. This means the leader-boards might be incompatible with the norms, thus causing stress in the work environment.

Table 5 summarises our findings regarding the personal and cultural values related to gamification and how it links to ethical issues.

Table 5. Gamification vs. personal and cultural values

\begin{tabular}{|l|l|l|}
\hline & Raise Ethical Issues & Likely to Reduce \\
\hline \multirow{2}{*}{ Value Sensitive Design } & Not-aligned with personal values & Aligned with personal values \\
\cline { 2 - 3 } & Forced to participate & Participation is an option \\
\hline \multirow{2}{*}{ Quality Standards } & Drive people to be fast & Quality first \\
\cline { 2 - 3 } & Create clear competition & Soft competition \\
\hline \multirow{2}{*}{ Honesty } & Difficult to win & Everyone can get something \\
\cline { 2 - 3 } & Consequences on losing & No serious consequences \\
\hline Culture of the Place & Incompatible & Compatible \\
\hline
\end{tabular}

\section{Conclusions and Future Work}

We have investigated the debate regarding the ethical issues that gamification could cause within enterprises. Gamification could be seen as an unfair mechanism to increase productivity with no real costs, i.e. via playfulness. In addition, it could increase pressure on employees to achieve more or avoid being in the bottom of the list. Gamification might contradict with some personality types and cultural norms. In our future work, we will explore these issues related to the ethical use of gamification, including its inter-relation with culture, personality traits, and managerial styles. As a social and moral responsibility, we will also try to standardise a code of ethics for software developers and enterprises who design, build and implement gamification.

Acknowledgements. The research was supported by an FP7 Marie Curie CIG grant (the SOCIAD Project) and by Bournemouth University through the Fusion Investment Fund and the Graduate School Santander Grant for PGR Development.

\section{References}

1. Deterding, S., Dixon, D., Khaled, R., Nacke, L.: From game design elements to gamefulness: defining gamification. In: International Academic MindTrek Conference: Envisioning Future Media Environments, pp. 9-15 (2011)

2. Webb, E.N., Cantú, A.: Building internal enthusiasm for gamification in your organization. In: Kurosu, M. (ed.) HCII/HCI 2013, Part II. LNCS, vol. 8005, pp. 316-322. Springer, Heidelberg (2013)

3. Huotari, K., Hamari, J.: Defining gamification: a service marketing perspective. In: Proceeding of the 16th International Academic MindTrek Conference, pp. 17-22 (2012)

4. Simoes, J., Redondo, R.D., Vilas, A.F.: A social gamification framework for a K-6 learning platform. Comput. Human Behav. 29, 345-353 (2013) 
5. O‘Donovan, S., Gain, J., Marais, P.: A case study in the gamification of a university-level games development course. In: Proceedings of the South African Institute for Computer Scientists and Information Technologists Conference on - SAICSIT 2013, p. 242. ACM Press, New York (2013)

6. Herzig, P., Ameling, M., Schill, A.: A Generic Platform for Enterprise Gamification. In: 2012 Joint Working IEEE/IFIP Conference on Software Architecture and European Conference on Software Architecture, pp. 219-223. IEEE (2012)

7. Volkswagon: Piano Staircase | The Fun Theory, http://www.thefuntheory.com/ piano-staircase

8. Creswell, J.W., Plano Clark, V.L.: Designing and conducting mixed methods research. SAGE Publications, Inc. (2011)

9. Myers, M.D., Newman, M.: The qualitative interview in IS research: Examining the craft. Inf. Organ. 17, 2-26 (2007)

10. Nicholson, S.: A User-Centered Theoretical Framework for Meaningful Gamification A Brief. Games+ Learning+ Society 8 (2012)

11. Friedman, B., et al.: Value sensitive design and information systems. In: Early Engagement and New technologies: Opening up the laboratory, pp. 55-95. Springer, Netherlands (2013) 